\title{
Thermal Radiation Effects on Heat and Mass Transfer of Magnetohydrodynamics Dusty Jeffrey Fluid Past an Exponentially Stretching Sheet
}

\author{
Siti Nur Haseela Izani and Anati Ali* \\ Department of Mathematical Sciences, Faculty of Science \\ Universiti Teknologi Malaysia, 81310 UTM Skudai, Johor, Malaysia \\ *Corresponding author: snhaseela15@gmail.com
}

Article history

Received: 23 March 2019

Received in revised form: 23 March 2019

Accepted: 21 May 2019

Published online: 1 August 2019

\begin{abstract}
The heat and mass transfer of steady magnetohydrodynamics of dusty Jeffrey fluid past an exponentially stretching sheet in the presence of thermal radiation have been investigated. The main purpose of this study is to conduct a detailed analysis of flow behaviour of suspended dust particles in non-Newtonian fluid. The governing equations hav been converted into dimensionless form, and then solved numerically via the Keller-box method. The expression of Sherwood number, Nusselt number and skin friction have been evaluated, and then displayed in tabular forms. Velocity, temperature and concentration profiles are presented graphically. It is observed that large value of dust particles mass concentration parameter has reduced the flow velocity significantly. Increase in radiation parameter enhances the temperature, whereas the increment in Schmidt number parameter reduces the concentration.
\end{abstract}

Keywords Dusty Jeffrey fluid; two-phase flow; heat and mass transfer; thermal radiation; particle-fluid interaction.

Mathematics Subject Classification 76T10, 76W05, 80A20

\section{Introduction}

Multiphase flows (either two or more phases) are described as the flows of different types of matter. Physically, they represent carrier phase consisting of particulate phase of any randomly chemical component. The carrier phase is either gas, liquid or solid. Stability of the laminar flow of a dusty gas was first investigated by Saffman [1], in which the author found that the two-phase fluids flow is the simplest model of multiphase flow. These phase combinations could be liquid-solid flow, gas-solid flow, liquid-gas flow, or liquid-liquid flow. Soo [2] discovered that in most cases, when the carrier phase is a gas, the particulate phase may consist of solid particles or liquid droplets, or both. Meanwhile, when it comes to liquid as a carrier phase, the particulate phase may consist of solid particles, gas, or immiscible liquid components. 
The interest in studying the flow of fluids with suspended solid particles has gained much attention nowadays due to their usage potential in industrial and technological processes. Some common examples of applications of multiphase fluids in various industrial fields are environmental pollutant motions, filtration, spray processes, fluid-particle transport, pollution control, foodstuff processing, groundwater flow, nuclear reactor cooling, and drug aerosol delivery. Wide application of fluid flow with dust particles in the engineering field includes airlift pump, aerodynamics ablation, paint spraying, dust collectors, atmospheric fallout, powder technology, and rain erosion. In last few decades, Sproull [3] experimentally studied air flow motion consisting of small dust particles. Tyndall [4] observed that a particle free zone around a hot surface would appear in dusty air. Due to these reasons, dusty fluid flow, which is exclusively related to Saffman's model, has received special attention among researchers such as Marble [5], Nayfeh [6], Drew [7] and Siddiqa et al. [8]. Later on, study on boundary layer for dusty fluid was well written by Singleton [9].

Analyses on non-Newtonian dusty fluid flow in various aspects from both analytical and numerical methods have increased greatly, majorly due to the rapid development in many industrial applications, especially in polymer and food industries. For instance, some foodstuffs utilize fluids that exhibit the non-Newtonian behaviour, which promotes flow characteristic of non-Newtonian dusty fluid significantly.

Arifin et al. [10] reported the influence of aligned magnetic field and Newtonian heating on dusty Casson fluid over a stretching sheet, in which they found that Casson parameter led to the increase of skin friction coefficient and decrease of Nusselt number. Siddiqa et al. [11] analyzed the natural convection flow of dusty non-Newtonian fluid via a modified power-law viscosity model. Afterwards, Mahanthesh and Gireesha [12] studied dusty Casson fluid in the presence of thermal Marangoni convection effects. In both articles, the authors revealed that the heat transfer rate for dusty non-Newtonian fluid was higher as compared to dusty Newtonian fluid.

Makinde et al. [13] conducted numerical analysis on non-linear thermal radiation on magnetohydrodynamic dusty micropolar fluid. Very recently, Siddiqa et al. [14] studied dusty Casson fluid in the presence of radiative heat transfer, in which they observed that the radiation parameter rose along with the rate of heat transfer extensively. They concluded that the use of the non-Newtonian parameter can decrease drag friction and boost temperature profile in related engineering fields.

Various studies on non-Newtonian fluid with solid dust particles have shown that most researchers limited their interest to study heat transfer boundary layer flow only. In addition, most of the studies above only reported about non-Newtonian fluids such as the Casson fluid, modified power-law fluid and micropolar fluid. As diversification, research on this type of fluid can be extended to different models. One of them is Jeffrey fluid type model, which exhibits both viscous and elastic characteristics. In this regard, Bhatti and Zeeshan [15] studied the effects of variable viscosity on dust phase in unsteady dusty Jeffrey fluid flow; however, the author did not consider the mass transfer phenomena. In that article, the solution was obtained analytically. Thus, in present work, mass transfer has been included into the study on dusty Jeffrey fluid flow, with consideration on radiation effect. To the best of author's knowledge, no attempt has been reported on numerical analysis of steady two-phase dusty non-Newtonian fluid flow by using Jeffrey fluid model under influence of heat and mass transfer. The governing equations with boundary condition have been reduced to dimensionless form by employing dimensionless variables. The equations are then solved numerically with the help of Keller-box method. The 
expressions of Sherwood number, Nusselt number and skin friction have been evaluated and displayed in tabular forms. Velocity, temperature and concentration profiles have been analyzed based on their graphical behaviours, respectively, while suspended solid dust particle on Jeffrey fluid flow, together with heat and mass transfer characteristics, have been analyzed in detail.

\section{Mathematical Formulation}

For this case, steady two-dimensional mixed convection flow of dusty Jeffrey fluid over an exponentially stretching sheet has been considered. Heat and mass transfer under the influence of thermal radiation are assumed to take place in the flow. The velocity of the exponential stretching sheet is in the form $U_{w}(x)=U_{0} e^{x / L}$ ( $U_{0}$ being characteristic fluid velocity). The heating of the carrier fluid or cooling surface is maintained by temperature $T_{w}(x)=T_{\infty}+\left(T_{0}-\right.$ $\left.T_{\infty}\right) e^{x / 2 L}$ and exponential concentration $C_{w}(x)=C_{\infty}+\left(C_{0}-C_{\infty}\right) e^{x / 2 L}\left(U_{0}, T_{0}\right.$ and $C_{0}$ are positive constants). $L$ is reference length and subscripts $\infty, w$ are free stream conditions and stretching surface, respectively.

\subsection{Physical Assumption for (Solid) Dust Particles}

Following Marble [16], some assumptions have been made for dilute suspensions as below:

1. The dust particles have spherical shape. They are all rigid and have the same mass $m$ and radius $r$.

2. Small spherical dust particles of velocity $u_{p}$ move parallel through the carrier fluid at a small velocity $u$. Thus, the Reynold number for each dust particle is assumed to be small enough.

3. The dust particles are considered as non-interacting (being uniformly distributed throughout the carrier fluid).

4. The volume fraction of the dust particle $\phi$ is neglected due to some reasons.

Under this restriction, the total fluid-particle interaction force per unit volume $\mathbf{F}_{D}$ is needed along with the momentum equation. Keeping in mind, the motion of carrier fluid containing solid particles is critically dependent upon the interaction between the fluid and particle. The expression of $\mathbf{F}_{D}$ in vector form that obeys the Stokes law is

$$
\mathbf{F}_{D}=K N\left(\mathbf{V}_{p}-\mathbf{V}\right)
$$

where $K=6 \pi r \mu$ is the drag coefficient of each spherical dust particle, $N=\rho_{p} / m$ is the number density of particles. $\mu, \rho_{p}, \mathbf{V}=(u, v)$ and $\mathbf{V}_{p}=\left(u_{p}, v_{p}\right)$ are the fluid viscosity, dust particle density and velocity vector for fluid and dust particles, respectively. Similarly, $Q_{p}=\rho_{p} c_{p}\left(T_{p}-T\right) / \tau_{T}$ corresponding to the total heat transfer per unit volume is required for both phases, along with the energy equation. Here, $c_{p}, T_{p}, T, \tau_{T}$ are considered as specific heat of the dust particle, dust temperature, fluid temperature and the timescale for a particle to relax to the same temperature as the fluid flow. Detailed work on the interaction between fluid and particle can be found in the book by Rudinger [17] and Fan and Zhu [18]. 


\subsection{Constitutive Equation for a Jeffrey Fluid}

Following Qasim [19], the satisfying constitutive equation for a Jeffrey fluid is defined as

$$
\boldsymbol{\tau}=-p \boldsymbol{I}+\boldsymbol{S},
$$

where $\boldsymbol{\tau}$ is the Cauchy stress tensor, $p$ is the scalar pressure, and $\boldsymbol{I}$ is the identity tensor. The extra stress tensor, $\boldsymbol{S}$ is given as

$$
\boldsymbol{S}=\frac{\mu}{1+\lambda_{1}}\left(\boldsymbol{R}_{\mathbf{1}}+\lambda_{2}(\boldsymbol{V} \cdot \nabla) \boldsymbol{R}_{\mathbf{1}}\right),
$$

and

$$
\boldsymbol{R}_{1}=\nabla \boldsymbol{V}+(\nabla \boldsymbol{V})^{t}
$$

Here, $\lambda_{1}$ and $\lambda_{2}$ are the material parameters of Jeffrey fluid, $\boldsymbol{R}_{\mathbf{1}}$ is the first Rivlin-Eriksen tensor and $t$ denotes the matrix transpose. To clarify, if $\boldsymbol{F}_{D}=\lambda_{1}=\lambda_{2}=0$, it will lead to the expression of a single phase of an incompressible viscous flow.

\section{Governing Equations}

Under the above assumptions, the governing equations of heat and mass transfer for a steady flow of dusty Jeffrey fluid, in the presence of thermal radiation, can be written in dimensional form as

For Jeffrey fluid phase:

$$
\begin{gathered}
\frac{\partial u}{\partial x}+\frac{\partial v}{\partial y}=0 \\
u \frac{\partial u}{\partial x}+v \frac{\partial v}{\partial y}=\frac{v}{1+\lambda_{1}}\left[\frac{\partial^{2} u}{\partial y^{2}}+\lambda_{2}\left(u \frac{\partial^{3} u}{\partial x \partial y^{2}}+v \frac{\partial^{3} u}{\partial y^{3}}-\frac{\partial u}{\partial x} \frac{\partial^{2} u}{\partial y^{2}}+\frac{\partial u}{\partial y} \frac{\partial^{2} u}{\partial x \partial y}\right)\right] \\
+\underbrace{\frac{K N}{\rho}\left(u_{p}-u\right)}_{\text {extra force }}-\frac{\sigma B^{2} u}{\rho}, \\
u \frac{\partial T}{\partial x}+v \frac{\partial T}{\partial y}=\frac{\kappa}{\rho c_{f}} \frac{\partial^{2} T}{\partial y^{2}}+\frac{\rho_{p} c_{p}}{\rho c_{f} \tau_{T}}\left(T_{p}-T\right)+\frac{\rho_{p}}{\rho c_{f} \tau_{v}}\left(u_{p}-u\right)^{2}- \\
u \frac{\partial C}{\partial x}+v \frac{\partial C}{\partial y}=D_{m} \frac{\partial^{2} C}{\partial y^{2}}+\frac{\rho_{p}}{\rho \tau_{c}}\left(C_{p}-C\right) .
\end{gathered}
$$

For dust particle phase:

$$
\begin{gathered}
\frac{\partial u_{p}}{\partial x}+\frac{\partial v_{p}}{\partial y}=0, \\
u_{p} \frac{\partial u_{p}}{\partial x}+v_{p} \frac{\partial u_{p}}{\partial y}=-\frac{K}{m}\left(u_{p}-u\right), \\
u_{p} \frac{\partial T_{p}}{\partial x}+v_{p} \frac{\partial T_{p}}{\partial y}=-\frac{1}{\tau_{T}}\left(T_{p}-T\right),
\end{gathered}
$$




$$
u_{p} \frac{\partial C_{p}}{\partial x}+v_{p} \frac{\partial C_{p}}{\partial y}=\frac{1}{\tau_{c}}\left(C-C_{p}\right)
$$

subject to the boundary conditions

$$
\begin{gathered}
u=U_{w}(x), \quad v=0, \quad T=T_{w} \quad C=C_{w} \quad \text { at } y=0 \\
u \rightarrow 0, u_{p} \rightarrow 0, \quad v_{p} \rightarrow v, T \rightarrow T_{\infty}, T_{p} \rightarrow T_{\infty}, C \rightarrow C_{\infty}, \quad C_{p} \rightarrow C_{\infty} \text { as } y \rightarrow \infty
\end{gathered}
$$

where $v=\mu / \rho, \kappa, \rho, T, C, \sigma, c_{f}$ are the kinematic viscosity, thermal conductivity, density, temperature, concentration, electrical conductivity and specific heat of the fluid phase, respectively. $q_{r}$ is the radiative heat flux, $D_{m}$ is the diffusion coefficient, $C_{p}$ is the concentration for the dust particle phase, and $\tau_{c} \quad\left(\tau_{v}=m / K\right)$ is the timescale for each particle to relax to the same concentration (velocity) as the fluid flow. Since the effects of thermal radiation and magnetic field are taken into accounts, both variables need to be defined well in order to simplify a similarity solution. Referring to Rosseland's approximation, the radiative heat flux $q_{r}$ can be expressed as

$$
q_{r}=-\frac{4 \sigma^{*}}{3 k^{*}} \frac{\partial T^{4}}{\partial y}
$$

where $\sigma^{*}$ is the Stefan-Boltzmann and $k^{*}$ is mean absorption coefficient. $T^{4}$ may be identified as a linear function of temperature $T^{4} \equiv 4 T_{\infty}^{3} T-3 T_{\infty}^{4}$ due to the assumption that the temperature difference within the flow is small enough. The new expression of the radiative heat flux is derived as

$$
\frac{\partial q_{r}}{\partial y}=-\frac{16 \sigma^{*} T_{\infty}^{3}}{3 k^{*}} \frac{\partial^{2} T}{\partial y^{2}}
$$

Meanwhile, a magnetic field $B(x)=B_{0} e^{x / 2 L}$ is applied with $\operatorname{constant} B_{0}$.

\section{Similarity Transformation}

Now, the nonlinear governing equations are transformed into nonlinear ordinary differential equations via suitable transformations. The suitable transformations for both phases are

Fluid phase :

$$
u=U_{o} e^{x / L} f^{\prime}(\eta), v=-\sqrt{\frac{U_{o} v}{2 L}} e^{x / 2 L}\left[f(\eta)+\eta f^{\prime}(\eta)\right], \quad \theta(\eta)=\frac{T-T_{\infty}}{T_{w}-T_{\infty}}, \phi(\eta)=\frac{C-C_{\infty}}{C_{w}-C_{\infty}} .
$$

Dust phase :

$$
u_{p}=U_{o} e^{x / L} F^{\prime}(\eta), v_{p}=-\sqrt{\frac{U_{o} v}{2 L}} e^{x / 2 L}\left[F(\eta)+\eta F^{\prime}(\eta)\right], \theta_{p}(\eta)=\frac{T_{p}-T_{\infty}}{T_{w}-T_{\infty}}, \phi_{p}(\eta)=\frac{C-C_{\infty}}{C_{w}-C_{\infty}}
$$

with a dimensionless similarity variable

$$
\eta=y \sqrt{\frac{U_{o}}{2 v L}} e^{x / 2 L}
$$

where $\left(f^{\prime}(\eta), F^{\prime}(\eta)\right),\left(\theta(\eta), \theta_{p}(\eta)\right)$ and $\left(\phi(\eta), \phi_{p}(\eta)\right)$ are the dimensionless stream function, temperature and concentration of fluid and dust particle phase, respectively. The next step is 
introducing relation (16) into Equations (8)-(12), where the dimensionless nonlinear ordinary differential equations are obtained as

$$
\begin{gathered}
f^{\prime \prime \prime}+\left(1+\lambda_{1}\right)\left(f f^{\prime \prime}-2 f^{2}\right)+D e\left[2 f^{\prime} f^{\prime \prime \prime}-f f^{\prime \prime \prime \prime}+3\left(f^{\prime \prime}\right)^{2}\right]+2\left(1+\lambda_{1}\right) G \beta_{v}\left(F^{\prime}-f^{\prime}\right) \\
-\left(1+\lambda_{1}\right) M f^{\prime}=0 \\
(1+4 / 3 T R) \theta^{\prime \prime}+\operatorname{Pr}\left(f \theta^{\prime}-f^{\prime} \theta\right)+2 \operatorname{Pr} G \gamma \beta_{T}\left(\theta_{p}-\theta\right)+2 \operatorname{Pr} E c G \beta_{v}\left(F^{\prime}-f^{\prime}\right)^{2}=0 \\
2\left(F^{\prime}\right)^{2}-F F^{\prime \prime}+2 \beta_{v}\left(F^{\prime}-f^{\prime}\right)=0 \\
F^{\prime} \theta_{p}-F \theta_{p}^{\prime}+2 \beta_{T}\left(\theta_{p}-\theta\right)=0 \\
\phi^{\prime \prime}+S c\left(f \phi^{\prime}-f^{\prime} \phi\right)+2 S c G \beta_{c}\left(\phi_{p}-\phi\right)=0 \\
F^{\prime} \phi_{p}-F \phi_{p}^{\prime}+2 \beta_{c}\left(\phi_{p}-\phi\right)=0 .
\end{gathered}
$$

Accordingly, the transformed boundary conditions become

$$
\begin{aligned}
& f(0)=0, \quad f^{\prime}(0)=1, \quad \theta(0)=1, \quad \phi(0)=1 \quad \text { at } \quad \eta=0, \\
& \left.\begin{array}{r}
f^{\prime}(\eta) \rightarrow 0 \quad F^{\prime \prime}(\eta) \rightarrow 0, \quad F^{\prime}(\eta) \rightarrow f^{\prime}(\eta) \\
\theta(\eta) \rightarrow 0 \quad \theta_{p}(\eta) \rightarrow 0, \quad \phi(\eta) \rightarrow 0 \quad \phi_{p}(\eta) \rightarrow 0 \quad \phi_{p}(\eta) \rightarrow 0
\end{array}\right\} \text { as } \eta \rightarrow \infty
\end{aligned}
$$

$D e, G, \beta_{v}, M, T R, \operatorname{Pr}, \gamma, \beta_{T}, E c, S c$ and $\beta_{c}$ are Deborah number, dust particles mass concentration parameter, local fluid-particle interaction parameter for velocity, magnetic parameter, radiation parameter, Prandtl number, specific heat ratio, local fluid-particle interaction parameter for temperature, Eckert number, Schmidt number, and local fluid-particle interaction parameter for concentration, respectively, defined as

$$
\begin{aligned}
& D e=\frac{\lambda_{2} U_{0} e^{x / L}}{L}, \quad G=\frac{m N}{\rho}, \quad \beta_{v}=\frac{L}{\tau_{v} U_{0}}, \quad M=\frac{2 \sigma B_{0}^{2} L}{\rho U_{0}}, \quad \operatorname{Pr}=\frac{\mu c_{f}}{\kappa} \\
& \gamma=\frac{c_{p}}{c_{f}}, \quad \beta_{T}=\frac{L}{\tau_{T} U_{0}}, \quad E c=\frac{U_{0}^{2} e^{2 x / L}}{c_{p}\left(T_{w}-T_{\infty}\right)}, \quad S c=\frac{v}{D_{m}}, \quad \beta_{c}=\frac{L}{\tau_{c} U_{0}} .
\end{aligned}
$$

\section{$5 \quad$ Numerical Scheme}

The governing partial differential equations of flow, heat and mass transfer ((5) to (12)) are converted into a system of nonlinear ordinary differential equation ((17) to (22)) with the transformed boundary condition (23) by using similarity transformation. The equations are then solved numerically by using finite difference scheme known as Keller-box method. This method consists of a four-step approach to successfully obtain a numerical solution, which can be found in the study by Cebeci and Bradshaw [20]:

1. Firstly is to choose suitable substitutions as an unknown function, in order to convert the nonlinear governing equations to a first-order differential equation system. 
2. Domain discretization: To approximate the first order system, centred-difference scheme about mid-point is applied.

3. Newton's method: To linearize the resulting system of equations, Newton's method is used to express them in matrix-vector form.

4. Block tri-diagonal: To solve the linear system for both phases, formation of block tri-diagonal elimination technique is required.

To this end, the present results have been computed, as displayed in tables in the next section.

\section{Result and Discussion}

Heat and mass transfer of steady magnetohydrodynamics (MHD) of dusty Jeffrey fluid over an exponentially stretching sheet with the thermal radiation effects had been investigated numerically via Keller-box method, whose results in terms of skin friction coefficient $f^{\prime \prime}(0)$, heat transfer coefficient $\theta^{\prime}(0)$ and mass transfer coefficient $\phi^{\prime}(0)$ are presented in Tables $3-5$. Meanwhile, the influence of physical parameters for fluid and dust particles phase on the velocity $f^{\prime}(\eta), F^{\prime}(\eta)$, temperature $\theta(\eta), \theta_{p}(\eta)$ and species concentration profile $\phi(\eta), \phi_{p}(\eta)$ are displayed graphically in Figures 1 - 6.

Keller-box finite difference scheme had been used to obtain the numerical solutions. Hence, the robustness of the numerical method needed to be reported beforehand. For this purpose, the accuracy of the Keller-box method had been investigated, for comparison with methods in published works such as homotopy analysis method (HAM) obtained by Nadeem et al. [21], Keller-box method by Bidin and Nazar [22], shooting method by Chaudhary et al. [23], and Runge-Kutta-Felhberg integration scheme by Kameswaran et al. [24]. The numerical results had been compared with the earlier published results for some limiting cases. Note that, by putting $D e=\lambda_{1}=0$ in Equation (17), the solution can be reduced to dusty viscous fluid (represent Newtonian fluid study), similar to $G=\beta_{v}=\beta_{T}=\beta_{c}=0$ in Equations (18)-(22) which can be reduced to classical single-phase flow.

The comparison of the heat transfer coefficient by various values of $\mathrm{Pr}$ is presented in Table 1. The solution of Equation (17) in the present study can be reduced to single-phase problem (in the absence of solid dust particles) if $G=0$. As a consequence, Equation (17) is identical to the published result obtained by [21] by the use of Equation (14), which clearly shows the significance of single-phase problem (Jeffrey fluid model). Table 1 shows that heat transfer coefficient increased with an increase in the radiation parameter. The result is found to be in an excellent agreement between analytical solution [21] and numerical solution [22] when no radiation $T R=0$ and with radiation $T R=1$ cases were considered. Hence, this fact confirms the accuracy of method used.

Meanwhile, Table 2 shows the comparison of skin friction coefficient by different values of $M$. It can be seen that an increase in magnetic parameters led to the increase in the value of skin friction coefficient of fluid flow. Thus, the results are in good agreement with the results obtained by Chaudhary et al. [23] and Kameswaran et al. [24] 
Table 1: Comparison of Heat Transfer Coefficient $-\theta^{\prime}(0)$ between Various Values of $\operatorname{Pr}$ and $T R=0,1.0$ with $D e=G=\lambda_{1}=S c=0$

\begin{tabular}{|ccccc|}
\hline \multicolumn{5}{|c|}{$-\theta^{\prime}(0)$} \\
\hline$T R$ & $P r$ & Nadeem et al. $[21]$ & Bidin and Nazar $[22]$ & Present results \\
\hline \multirow{3}{*}{0} & 1.0 & 0.955 & 0.955 & 0.955 \\
& 2.0 & 1.471 & 1.471 & 1.471 \\
& 3.0 & 1.870 & 1.869 & 1.869 \\
& 1.0 & 0.534 & 0.532 & 0.532 \\
1 & 2.0 & 0.863 & 0.863 & 0.863 \\
& 3.0 & 1.121 & 1.121 & 1.121 \\
\hline
\end{tabular}

Table 2: Comparison of Skin Friction Coefficient $-f^{\prime \prime}(0)$ between Various Values of $M$ with $D e=G=\lambda_{1}=S c=T R=0$

\begin{tabular}{|ccccc|}
\hline \multicolumn{5}{|c|}{$-f^{\prime \prime}(0)$} \\
\hline$T R$ & $M$ & Chaudhary et al. $[23]$ & Kameswaran et al. $[24]$ & Present results \\
\hline & 0 & 1.282 & 1.282 & 1.282 \\
0 & 0.04 & 1.314 & - & 1.298 \\
& 0.25 & 1.464 & - & 1.378 \\
& 1.0 & - & 1.629 & 1.629 \\
& 2.0 & - & 1.913 & 1.913 \\
\hline
\end{tabular}

The values of skin friction coefficient $f^{\prime \prime}(0)$ by various values of magnetic parameter $M$, Deborah number $D e$ and fluid-particle interaction parameter for velocity $\beta_{v}$ are provided in Table 3. It can be seen that the values of skin friction coefficient increased with the increase of values of $M$ and $\beta_{v}$. Physically, the applied magnetic field in the fluid regime generated Lorentz force which gave rise to surface friction force. Put simply, one could improve the fluid flow rate by reducing the strength of the magnetic field, and vice versa. In addition, the presence of dust particle boosted more resistance to the flow, which enhanced the skin friction coefficient. In contrast to this, the influence of Deborah number $D e$ decreased the values of $f^{\prime \prime}(0)$ significantly.

Since the viscosity of Non-Newtonian fluid is higher compared to Newtonian fluid, this led to the increase of frictional forces created between two surfaces. Therefore, the viscosity of fluids might have affected the fluid flow. The same observation was reported in [13].

The effects on heat transfer coefficient $\theta^{\prime}(0)$ by various values of magnetic field $M$, Eckert number $E c$, radiation $T R$ and fluid-particle interaction parameter for temperature $\beta_{T}$ are shown in Table 4. It can be seen that the heat transfer coefficient increased with the increase of the values of $M$ and $\beta_{T}$. Physically, if the strength of the magnetic field rises, the rate of fluid motion will increase, which results in the enhancement of heat transfer coefficients. Also, it can be seen that the increase of the values of $E c$ and $T R$ reduced the heat transfer coefficient. The presence of Eckert number parameter generally created some thermal energy due to its frictional heating, and when thermal radiation parameter took place in the fluid regime, they significantly affected the temperature distribution and heat transfer. 
Table 3: Values of Skin Friction Coefficient $f^{\prime \prime}(0)$

\begin{tabular}{|c|c|c|c|}
\hline$M$ & $D e$ & $\beta_{v}$ & $f^{\prime \prime}(0)$ \\
\hline 1.0 & 0.5 & 5.0 & 3.491761 \\
3.0 & & & 3.684930 \\
6.0 & & & 3.956659 \\
& & & \\
& 2.0 & & 2.020731 \\
& 5.0 & & 1.321724 \\
& & & \\
& & 0.3 & 2.211438 \\
& & 100.0 & 3.825013 \\
\hline
\end{tabular}

Table 4: Values of Heat Transfer Coefficient $\theta^{\prime}(0)$

\begin{tabular}{|c|c|c|c|c|}
\hline$M$ & $E c$ & $T R$ & $\beta_{T}$ & $\theta^{\prime}(0)$ \\
\hline 1.0 & 2.0 & 0.5 & 5.0 & 2.916833 \\
3.0 & & & & 2.972564 \\
6.0 & & & & 3.037863 \\
& & & & \\
& 1.5 & & & 3.838100 \\
& 3.0 & & & \\
& & 1.074299 \\
& & 3.0 & & 2.105412 \\
& & & & 1.417124 \\
& & & 0.3 & 0.777226 \\
& & & 100.0 & 3.239598 \\
\hline
\end{tabular}

Table 5 displays the effect of the magnetic field $M$, Schmidt number $S c$ and fluid-particle interaction parameter for concentration $\beta_{c}$ on the mass transfer coefficient. The table shows that the mass transfer coefficient increased as the values of $S c$ and $\beta_{c}$ were increased. On the other hand, increasing the magnetic parameter obviously reduced the flow rate due to the existence of Lorentz force, which then reduced the mass transfer coefficient. However, it did not accelerate the species transfer in the boundary layer.

The effects of dust particle mass concentration parameter $G$ on the velocity profile for the carrier fluid system are depicted in Figure 1. From the graph, the presence of a small number of dust particle caused less resistance to the flow. However, the velocity of fluid decreased significantly according to the increase of number of particles. This behaviour was predictable, as the frictional forces between phases became stronger in the momentum boundary layer region.

Figure 2 and Figure 3 show the effects of magnetic field $M$ on velocity on concentration profiles for fluid and dust phases. It can be seen that the velocity of the fluid and dust phase in the boundary layer region decreased as the values of $M$ were increased, which was due to the fact that the Lorentz force was against the motion, hence it had tendency to delay the motion 
Table 5: Values of Sherwood Number $-\phi^{\prime}(0)$

\begin{tabular}{|c|c|c|c|}
\hline$M$ & $S c$ & $\beta_{c}$ & $\phi^{\prime}(0)$ \\
\hline 1.0 & 1.5 & 5.0 & 3.290832 \\
3.0 & & & 3.224334 \\
6.0 & & & 3.133396 \\
& & & \\
& 0.22 & & 3.037536 \\
& 2.80 & & 3.533988 \\
& & & \\
& & 0.3 & 2.172394 \\
& & 100.0 & 3.459989 \\
\hline
\end{tabular}

of fluid flow. Meanwhile, for the mass transfer problem, as $M$ was increased, concentration profile for both phases increased. This was also due to an increase in the opposing force known as Lorentz force. Once the magnetic field was applied to an electrically conducting fluid, the frictional heating between the fluids layers increased as well, releasing energy in the form of heat. Thus, it clearly indicates that Lorentz force could enhance the movement of species.

The effect of thermal radiation parameter, $T R$ on the temperature profile for fluid and dust particles phases, is presented in Figure 4. The temperature profiles of both phases increased as parameter $T R$ was increased. This temperature enhancement occurred due to energy being transmitted in the fluid; hence it obeys the definition of thermal radiation.

Viscous dissipation is commonly considered in non-Newtonian fluids studies. Eckert number is a non-dimension parameter that alludes to this effect. The energy yield by work done between fluid layers will be taken by fluid viscosity, which at the same time is transformed into internal energy of the fluid. Put simply, heat energy is provided when the fluid is heated up. This phenomenon is known as an irreversible process or termed as viscous dissipation. Therefore, in this case, the fluid temperature increased with the increase of values of $E c$. The influence of Eckert number on temperature profile is displayed in Figure 5.

Figure 6 depicts the influence of Schmidt number on the concentration profile. It can be seen that increasing the values of the Schmidt number reduced the concentration of fluid and dust particles in the boundary layer region. This behaviour was expected due to the increase in the kinematics viscosity which acted on the fluid flows. The Schmidt number is defined as the ratio of kinematic viscosity to the mass diffusivity. Hence, it is true that when the rate of mass transfer is dropped from the sheet to the fluid, it reduces the concentration of fluid and dust. The Schmidt number parameter, in fact, can be an excellent agent in controlling the movement of species.

\section{Conclusion}

1. The presence of a large number of dust particles in a carrier fluid phase offers more resistance to the flow.

2. Flow behaviour due to constrasting velocity and concentration of the magnetic parameter 


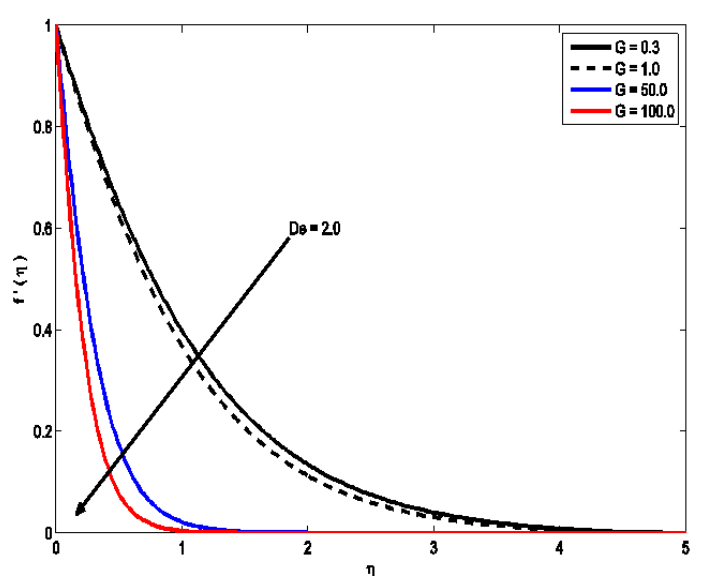

Figure 1: Velocity Profile of Different Values of $G$ on Dusty Jeffrey Fluid

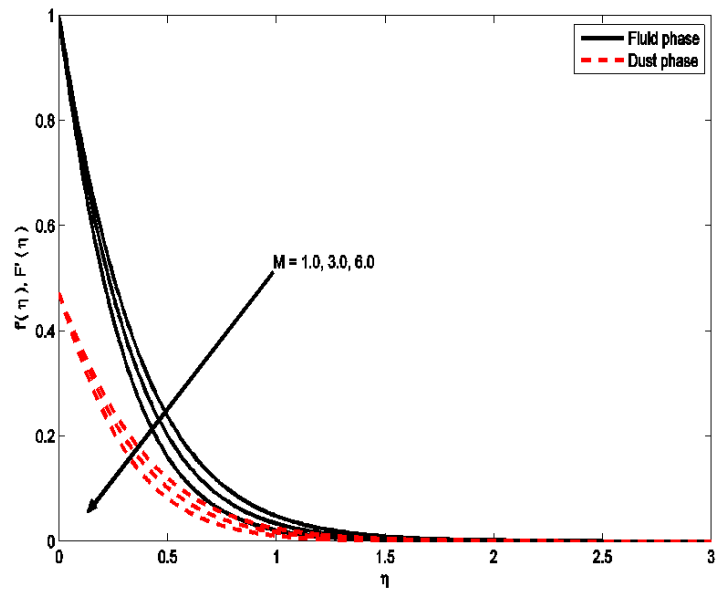

Figure 2: Effect of Magnetic Parameter $M$ on Velocity Profile

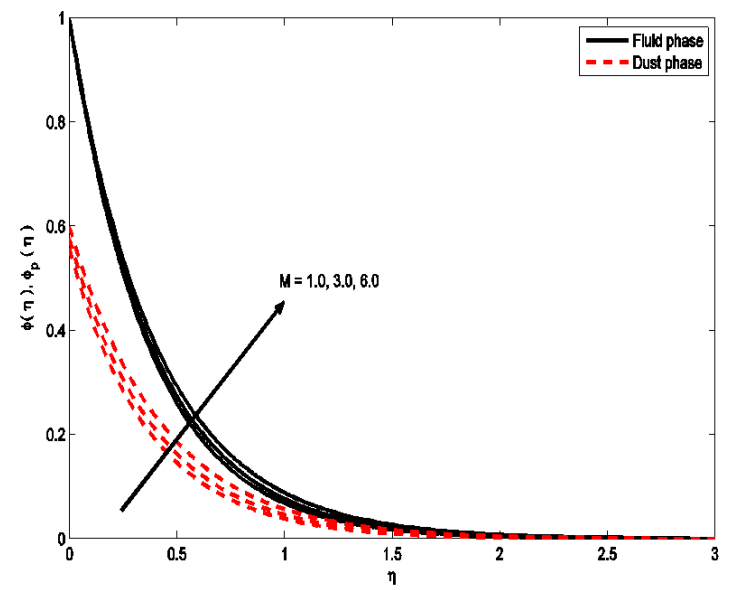

Figure 3: Effect of Magnetic Parameter $M$ on Concentration Profile 


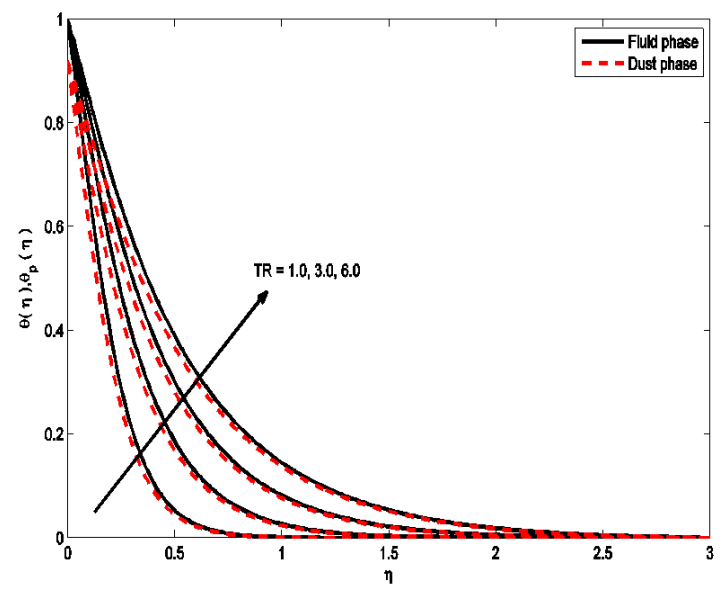

Figure 4: Effect of Radiation Parameter $T R$ on Temperature Profile

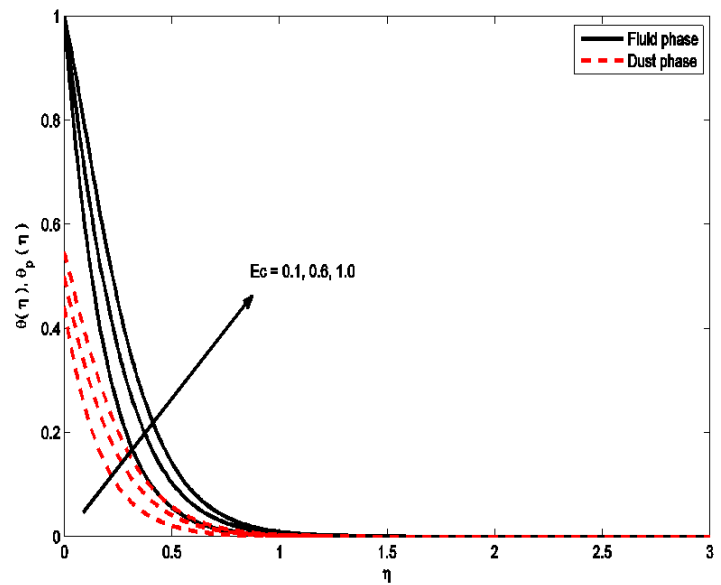

Figure 5: Effect of Eckert number $E c$ on Temperature Profile

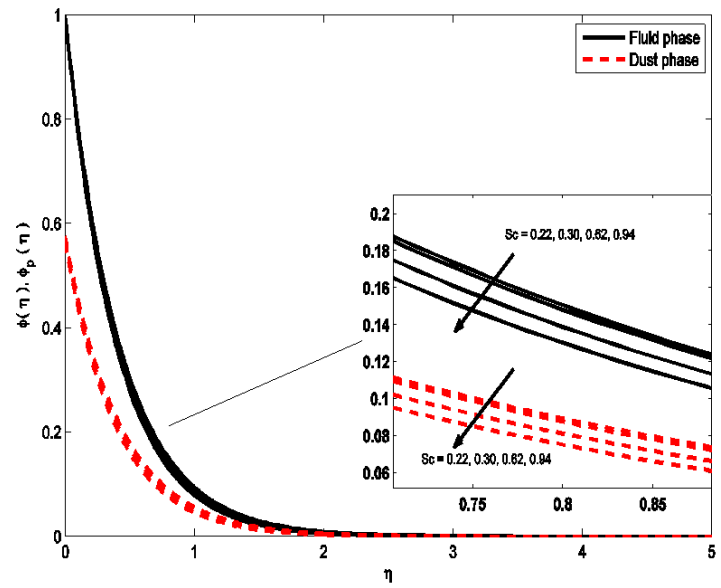

Figure 6: Effect of Schmidt Number $S c$ on Concentration Profile 
is opposite in both phases.

3. Increase in radiation and Eckert number parameters will enhance temperature in both phases.

4. Concentration distribution of fluid decreases with the increase of Schmidt number parameter.

\section{Acknowledgement}

The author would like to acknowledge the support from Ministry of Education Malaysia for awarding GE STEM grant (vote number 07379) and Research Management Centre-UTM, Universiti Teknologi Malaysia (UTM) for the financial support through vote number 13J80 for this research.

\section{References}

[1] Saffman, P. G. On the stability of laminar flow of a dusty gas. Journal of Fluid Mechanics. 1962. 13(1): 120-8.

[2] Soo, S. L. Multiphase Fluid Dynamics. Gower Technical Press. 1990.

[3] Sproull, W. T. Viscosity of dusty gases. Nature. 1961. 190(4780): 976.

[4] Tyndall, J. On dust and disease. Fraser's magazine. 1870. 1(3): 302-310.

[5] Marble, F. E. Dynamics of a gas containing small solid particles. Combustion and Propulsion, 5th AGARD Colloqium. Pergamon Press. 1963. 175-213.

[6] Nayfeh, A. H. Oscillating two-phase flow through a rigid pipe. AIAA Journal. 1966. 4(10): 1868-1870.

[7] Drew, D. A. Mathematical modeling of two-phase flow. Annual Review of Fluid Mechanics. 1983. 15(1): 261-291.

[8] Siddiqa, S., Hossain, M. A. and Saha, S. C. Two-phase natural convection flow of a dusty fluid. International Journal of Numerical Methods for Heat \& Fluid Flow. 2015. 25(7): 1542-1556.

[9] Singleton, R. E. Fluid mechanics of gas-solid particle flow in boundary layers. Ph.D. Thesis. California Institute of Technology. 1964.

[10] Arifin, N. S., Zokri, S. M., Kasim, A. R. M., Salleh, M. Z., Yusoff, W. N. S. W., Mohammad, N. F. and Shafie, S. Aligned magnetic field on dusty Casson fluid over a stretching sheet with Newtonian heating. Malaysian Journal of Fundamental and Applied Sciences. 2017. 13(3): 245-248.

[11] Siddiqa, S., Begum, N., Hossain, M. A., and Gorla, R. S. R. Natural convection flow of a two-phase dusty non-Newtonian fluid along a vertical surface. International Journal of Heat and Mass Transfer. 2017. 113: 482-489.

[12] Mahanthesh, B. and Gireesha, B. J. Thermal Marangoni convection in two-phase flow of dusty Casson fluid. Results in Physics. 2018. 8: 537-544. 
[13] Makinde, O. D., Kumar, K. G., Manjunatha, S. and Gireesha, B. J. Effect of nonlinear thermal radiation on MHD boundary layer flow and melting heat transfer of micro-polar fluid over a stretching surface with fluid particles suspension. In Defect and Diffusion Forum. Trans Tech Publications. 2017. 378: 125-136.

[14] Siddiqa, S., Begum, N., Hossain, M. A., Shoaib, M. and Reddy Gorla, R. S. Radiative heat transfer analysis of non-Newtonian dusty Casson fluid flow along a complex wavy surface. Numerical Heat Transfer, Part A: Applications. 2018. 73(4): 209-221.

[15] Bhatti, M. M. and Zeeshan, A. Analytic study of heat transfer with variable viscosity on solid particle motion in dusty Jeffery fluid. Modern Physics Letters B. 2016. 30(16): 1650196.

[16] Marble, F. M. Dynamics of dusty gases. Annual Review of Fluid Mechanics. 1970. 2(1): 397-446.

[17] Rudinger, G. Fundamental of Gas-Particle Flow. Amsterdam: Elsevier Scientific Publishing Company. 1980.

[18] Fan, L. S. and Zhu, C. Principles of Gas-Solid Flows. Cambridge University Press. 2005.

[19] Qasim, M. Heat and mass transfer in a Jeffrey fluid over a stretching sheet with heat source/sink. Alexandria Engineering Journal. 2013. 52(4): 571-575.

[20] Cebeci, T., and Bradshaw, P. Physical and Computational Aspects of Convective Heat Transfer. $1^{\text {st }}$ Edition. New York: Springer New York. 1988.

[21] Nadeem, S., Zaheer, S. and Fang, T. Effects of thermal radiation on the boundary layer flow of a Jeffrey fluid over an exponentially stretching surface. Numerical Algorithms. 2011. 57(2): 187-205.

[22] Bidin, B. and Nazar, R. Numerical solution of the boundary layer flow over an exponentially stretching sheet with thermal radiation. Eur. J. Sci. Res. 2009. 33(4): 710-717.

[23] Chaudhary, S., Singh, S. and Chaudhary, S. Thermal radiation effects on MHD boundary layer flow over an exponentially stretching surface. Applied Mathematics. 2015. 6(02): 295.

[24] Kameswaran, P. K., Narayana, M., Sibanda, P. and Makanda, G. On radiation effects on hydromagnetic Newtonian liquid flow due to an exponentially stretching sheet. Boundary Value Problems. 2012. (1): 105. 